\title{
Türkiye Florası İçin Yeni Dianthus (Caryophyllaceae) Kayıtları
}

\section{Ergin HAMZAOĞLU1 ${ }^{\mathscr{P}}$ (D), Murat KOÇ2(D)}

${ }^{1}$ Gazi Üniversitesi, Gazi Eğitim Fakültesi, Matematik ve Fen Bilimleri Eğitimi Bölümü, 06500, Yenimahalle, Ankara, ${ }^{2}$ Ankara Yıldırım Beyazıt Üniversitesi, Halk Sağıllğı Enstitüsü, Geleneksel, Tamamlayıcı ve Entegratif Tıp Anabilim Dalı, 06010, Keçiören, Ankara, Türkiye ${ }^{1}$ https://orcid.org/0000-0001-6053-6796, ${ }^{2}$ https://orcid.org/0000-0002-0829-4571

$\triangle$ : erginhamzaoglu@yahoo.com

\section{ÖZET}

Ardahan'dan toplanan Dianthus azkurensis Sosn. ile Kayseri, Malatya ve Mersin'den toplanan D. orientalis Adams subsp. aphanoneurus Rech.f. Türkiye florası için yeni kayıt olarak tanıtıldı. Ayrıca, taksonların detaylı betimlemeleri, yayılışları ve resimleri verildi.

\section{New Dianthus (Caryophyllaceae) Records For The Flora Of Turkey}

\section{ABSTRACT}

Dianthus azkurensis Sosn. and D. orientalis Adams subsp. aphanoneurus Rech.f. that were collected from Ardahan and, Kayseri, Malatya and Mersin were given as new records for the flora of Turkey. In addition, detailed descriptions, distributions and illustrations of the taxa were provided.

\section{Araștırma Makalesi}

Makale Tarihçesi

Geliş Tarihi : 08.01.2019

Kabul Tarihi : 28.02.2019

Anahtar Kelimeler
Dianthus
Yeni kayıt
Türkiye

\section{Research Article}

Article History

Received : 08.01.2019

Accepted : 28.02.2019

\section{Keywords}

Dianthus

New record

Turkey

To Cite : Hamzaoğlu E, Koç M 2019. Türkiye Florası İçin Yeni Dianthus (Caryophyllaceae) Kayıtları. KSÜ Tarım ve Doğa Derg 22(3): 381-388. DOI: 10.18016/ksutarimdoga.vi.509956

\section{GİRIŞ̧}

Caryophyllaceae Juss., içerdiği yaklaşık 90 cins ve 2400 tür sayısı ile dünyanın en büyük dikotil familyaları arasında yer alır (Thorne ve Reveal, 2007). Yapraklarının karşılıklı, çiçek segmentlerinin 5 parçalı ve meyvesinin kapsül tipinde olması, familyanın birçok cinsinde gözlenen en belirgin özelliklerdir. Yaklaşık 300 tür içeren Dianthus L., familyanın en büyük ikinci cinsidir. Cinse ait türlerin büyük kısmı Avrupa, Asya ve Kuzey Afrika'da yetişir. $\mathrm{Bu}$ alanların kesiştiği bölgede yer alan Akdeniz, cinsin gen merkezi olarak kabul edilir (Reeve, 1967; Constantinidis, 1999; Dequan ve Turland, 2001; Mabberley, 2008; Valente ve ark., 2010; Vaezi ve ark., 2014). Dianthus, kaliksinin tüpsü ve epikaliks segmentleriyle çevrilmiş olması, tohumlarının peltat olması, petalinin limb ve kılav olarak belirgin kısımlara ayrilması ile akraba cinslerden kolayca ayrilır (Bittrich, 1993; Bacchetta ve ark., 2010).

Türkiye Dianthus cinsi taksonları ile ilgili ilk kapsamlı çalışma Boissier (1867) tarafindan yazılmış "Flora Orientalis" tir. Bu eserde verilen 89 Dianthus türünden 48’i Türkiye florası ile ilişkilidir. Eser sadece Türkiye ile ilgili olmamasina rağmen, Dianthus cinsinin o dönemde Türkiye'den bilinen tüm taksonlarını içermesi açından oldukça önemlidir. Boissier bu eserde Diantheae oymağı altında değerlendirdiği Dianthus cinsini Verruculosi, Leiopetali, Fimbriati, Dentati ve Carthusiani şeklinde 5 grup altında toplamıştır.

Editörlüğünü Davis'in yaptığ "Flora of Turkey and the East Aegean Islands" (Türkiye Florası) adlı eser ise, Dianthus cinsinin sadece Türkiye taksonlarına odaklanan ilk eserdir. Eserde Dianthus cinsini yazan Reeve (1967), Williams'in (1893) monografindan oldukça yararlanmıştır. Reeve revizyonda önce cinsin betimlemesini vermiş, ardından hibritleşme ve erkek çiçeklerin taksonomide neden olduğu zorluklardan ve yaptığı revizyonda dikkate aldığı taksonomik karakterlerden bahsetmiştir. Dianthus cinsini Silenoideae alt familyası altında değerlendiren Reeve, cinse ait 67 tür tanıtmıştır. Ayrıca, Boissier (1867) tarafindan önerilen isimleri kullanarak cinsi Verruculosi, Leiopetali, Fimbriati, Dentati ve Carthusiani olmak üzere 5 gruba ayırmıştır.

Dalcı (1986) tarafindan Doğu Anadolu Dianthus türleri üzerinde yapılan çalışma, hem cinsin taksonomisine yeni karakter katması ve hem de çok sayıda popülasyon incelenmesi bakımından önemlidir. Ancak Dalcı'nın çalışması Dianthus cinsinin sadece 
Doğu Anadolu Bölgesinde yayılış gösteren taksonları ile sınırlı kaldığından, diğer bölgelerdeki yakın akraba taksonlarla mukayese şansı olmamıştır. Son zamanlarda Türkiye'nin farklı yerlerinde yapılan çalışmalar sonucu çok sayıda Dianthus örneği toplanmıştır. Toplanan bu örneklerin detaylı incelenmesi sonucu yeni taksonlar tanımlanmıştır. Son çalışmalar sonucu, cinsin Türkiye'deki tür sayısı 81'e yükselmiştir (Reeve, 1967; Özhatay ve Kültür, 2006; İlçim ve ark., 2013; Hamzaoğlu ve Koç, 2015; Hamzaoğlu ve ark., 2014; 2015a; 2015b; 2015c; 2018).

\section{MATERYAL ve METOT}

$\mathrm{Bu}$ çalışmanın materyalini Türkiye'nin farklı illerinden toplanmış örnekler, GAZI, ANK ve HUB herbaryumunda bulunan örnekler ve $\mathrm{K}$ ve $\mathrm{E}$ sanal herbaryumlarında sergilenen taranmış resimler oluşturmaktadır. Taksonların habit ve çiçek resimleri Canon EOS 60D dijital kamera ile çekilmiştir. Taksonların geçerli isimleri, orijinal yayınları, tiplendirmeleri, betimlemeleri, çiçeklenme ayları ve habitatları verilmiştir. Betimlemeler yazılırken, uzunluk ölçülerinde $0.5 \mathrm{~mm}$ hassasiyetli cetvel kullanılmıştır.

\section{BULGULAR ve TARTIŞMA}

Bu çalışmada; daha önce Flora USSR'de yer alan Dianthus azkurensis Sosn. ile Flora Iranica'da yer alan Dianthus orientalis Adams subsp. aphanoneurus Rech.f. Türkiye florası için yeni kayıt olarak tanıtıldı (Schischkin, 1985; Rechinger, 1988). Ayrıca politipik tür olan $D$. orientalis'in tür altı taksonları için ayırım anahtarı ve yayılış bilgileri verildi.

Dianthus azkurensis Sosn., Vestn. Tiflissk. Bot. Sada n.s., 1: 74 (1923), Şekil 1.

Holotypus: [Gürcistan, Georgia] Between Akhaltsikh and Atskhur [Ahıska-Azgur arasi], Toplayıcı? (TBI?).

Çokyıllık, demetsi otlar. Gövdeler dik, 15-35 cm boyunda, dallanmış, altta havlı, üstte tüysüz, 4-7dügümlü. Kısır sürgün yaprakları az sayıda, şeritsi, yassı, gövde yapraklarından daha uzun. Alt yapraklar çiçekteyken kuruyucu/dökülücü; orta yapraklar şeritsi, $20-35 \times 1-1.5 \mathrm{~mm}$, kanalsı veya \pm yuvarlak, gövdeye basık, düğümarasından kısa, yumuşak, tüysüz, uç sivri-sipsivri, kın boyu eninin en fazla 2 katı kadar. Çiçekdurumu sıkı simöz, çiçekler dal ucunda tek; pediseller 5-40 mm. Kaliks pulları 4; en dıştakiler kıkırdaksı, samanrengi veya bazen uçta yeşilimsimorumsu, altta damarsız, üstte 3-5-damarl, tüysüz, ucu kaliksten ayrık, kaliksin 1/3-3/4'ü kadar, geniş tersyumurtamsi, $3.5-5.5(-8) \times 2.2-3.5 \mathrm{~mm}$, kenar c. 0.3 $\mathrm{mm}$ eninde dar zarlı, uç emarginat veya bazen kütkesik, mukrolu veya kılçıkll, mukro veya kılçık tüm pulun 1/4-1/3(-1/2)'si kadar; en içtekiler dıştakilere benzer, kaliksin 1/3-1/2'si kadar, 3.5-5 × 3-4 mm, mukrolu, mukro tüm pulun 1/4-1/3'ü kadar. Kaliks yumurtamsı veya mizraksı, 8.5-11 × 3-4 mm, 30-35damarll, tüysüz, yeşil veya bazen morumsu lekeli; dişler geniş üçgensi, 1.6-2.2 × 1.2-1.8 mm, belirgin 3damarlı, uç sivri veya sipsivri. Petal $12-16 \mathrm{~mm}$; aya \pm yuvarlak, 4-6 $\times 3.5-5.5 \mathrm{~mm}$, tüm petalin c. $2 / 5$ 'i kadar, c. 3/4'ü kaliksin dışında, beneksiz, ebarbulat, üst beyaz, alt kremsi-yeşil, bazen uçta pembemsi, uç düz; kılav 8-10 × 1-1.3 mm, yaka eni kılav eninin c. 1/2'si kadar. Meyve açıkça kaliksten daha uzun. Tohumlar geniş eliptik, siyah, 1.5-2.2 x 1.2-1.5 mm. Ciçeklenme: Haziran-Temmuz. Habitat: Kayalık otlu yerler, 1950 $m$.

Avrupa-Sibirya elementi. Gürcistan, Türkiye. A9 Ardahan: Çıldır, Yıldırımtepe köyü, Şeytankalesi civarı, 1950 m, kayalık otlu yerler, 4.7.2013, Hamzaoğlu 6815 \& Koç (GAZI!, ANK!, HUB!).

Dianthus azkurensis, Gürcistan'da Ahıska (Akhaltsikh) ile Azgur (Atskhur) arasından bilinen bir türdür (Schischkin, 1985).
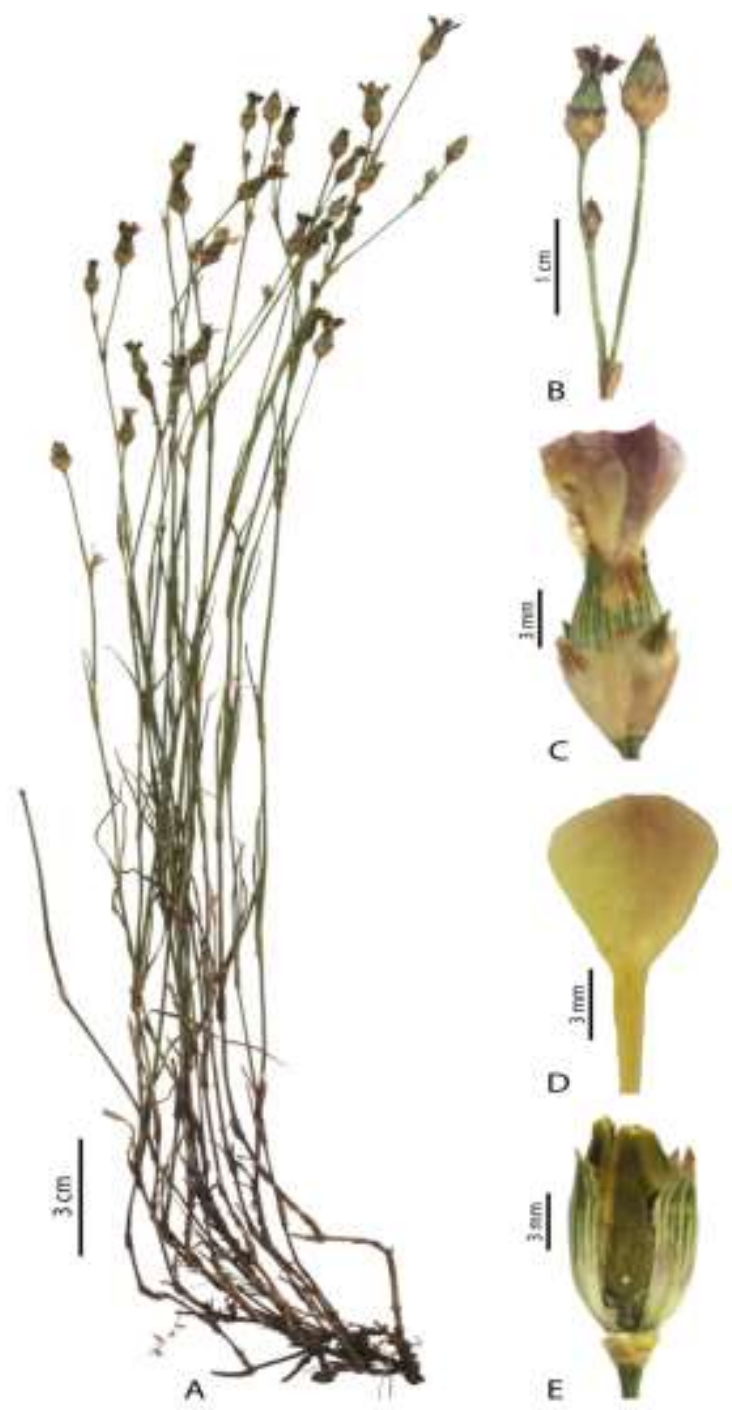

Şekil 1. Dianthus azkurensis. A- Habit, BÇiçekdurumu, C- Çiçek, D- Petal, E- Meyve. 

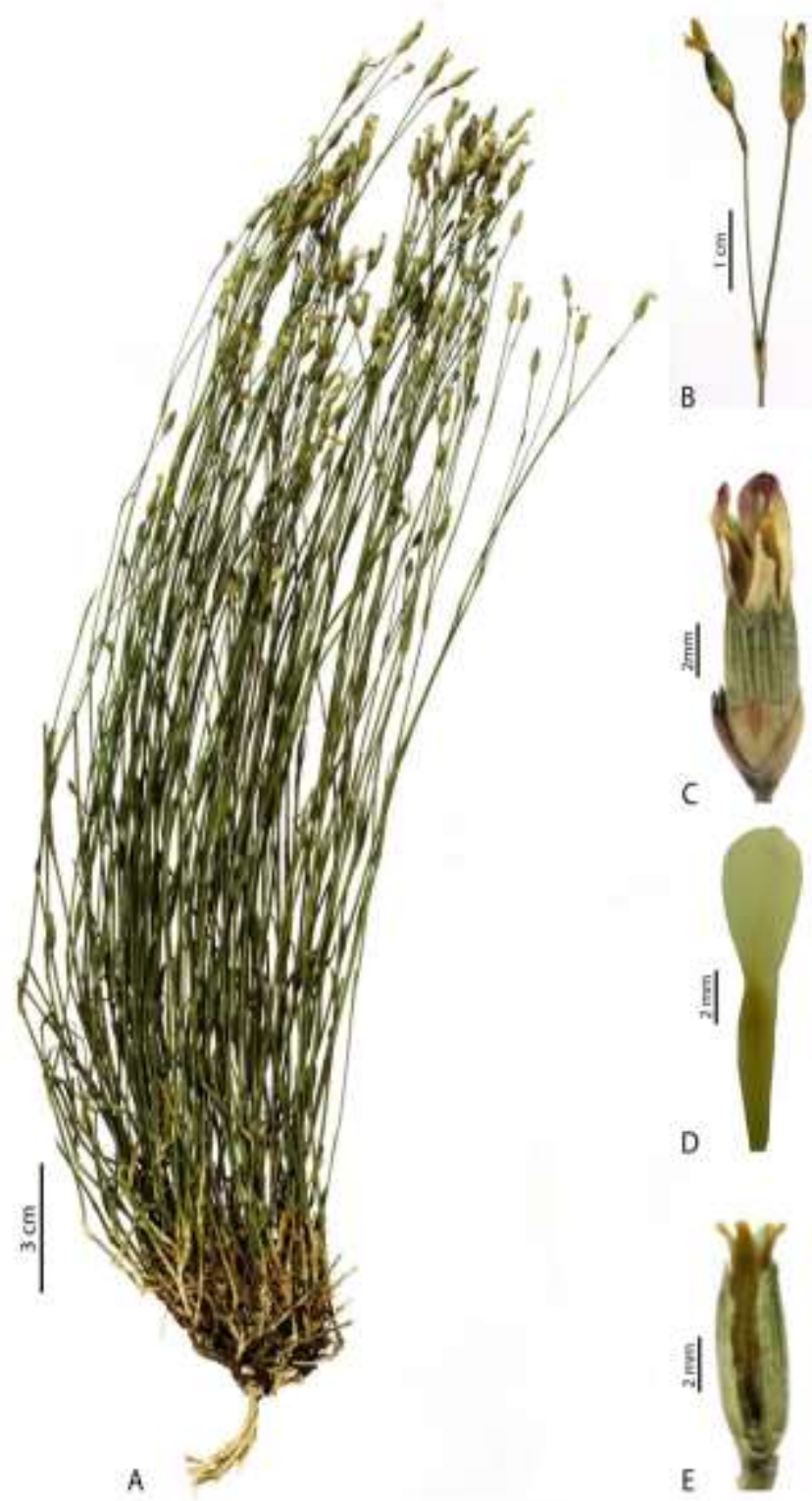

Şekil 2. Dianthus micranthus. A- Habit, BÇiçekdurumu, C- Çiçek, D- Petal, E- Meyve
Çıldır (Ardahan) adresi ile Ahıska adresi arasında kuş bakışı yaklaşık $70 \mathrm{~km}$ vardır. Türün Türkiye florasındaki en yakın akrabası Anadolu'nun güneyi için endemik olan Dianthus micranthus'tur (Reeve, 1967). Habit olarak benzemelerine rağmen, bu iki tür arasında belirgin taksonomik farklar vardır (Şekil 2-3, Çizelge 1).

Dianthus orientalis Adams, Beitr. Naturk., 1: 54 (1805).

Yarıçalımsı, sık demetsi otlar. Gövdeler dik veya eğik, 10-45 cm boyunda, bazen dallanmış, tüysüz veya havlı, 3-11-düğümlü. Kısır sürgün yaprakları şeritsi veya ipliksi-şeritsi, yassı veya kanalsı, gövde yapraklarına eşit veya en fazla 1.5 katı kadar. Alt yapraklar çiçeklenmede kalıcı veya kuruyucu/dökülücü; orta yapraklar şeritsi veya ipliksi-şeritsi, 10-50 × 0.5-2.3 $\mathrm{mm}$, yassı veya kanalsı, gövdeye basık veya \pm ayrık, düğümarasından kısa veya uzun, tüysüz, uç sivri veya sipsivri, kın boyu eninin 1-3 katı kadar. Çiçekdurumu simöz, gövdeler tek veya bazen birkaç çiçekli, çiçekler dal ucunda tek; pediseller 5-45 mm. Kaliks pulları (46-12(-16), sıkı ve düzenli veya gevşek ve düzensiz kiremitsi dizilmiş; en dıştakiler otsu veya kıkırdaksı, yeşilimsi, samanrengi veya morumsu, tabanda damarsız, uca doğru silik 1-7-damarlı, tüysüz, kaliksin 1/8-4/5’i kadar, şeritsi, mızraksı veya eliptik, 3-14 $\times$ 0.6-3 mm, kenar 0.2-0.3 mm eninde dar zarsı, uç sivri, kılçıklı, kuspisli veya mukrolu, şayet varsa kılçık tüm pulun 1/2-2/3'ü kadar; en içtekiler dıştakilere benzer, uca doğru silik 5-15-damarll, kaliksin 1/3-1/2'si kadar, yumurtamsı, tersmızraksı veya paralel-mizraksı, 7-15 $\times$ 2.2-3.8 mm, kenar 0.3-0.6 mm eninde zarsı, uç kütsivri veya küt-kesik, kılçıklı veya kuspisli, kılçık veya kuspis tüm pulun $1 / 5-1 / 3$ '̈ kadar.

Kaliks silindirik-mızraksı, $16-30 \times 2-4 \mathrm{~mm}$, genellikle belirgin 35-45-damarlı, tüysüz, samanrengi, yeşilimsi veya morumsu; dişler dar üçgensi-mızraksı, 5-9(-11) $\times$ 1-2 mm, tüysüz, 3-11-damarl, uç sivri veya sipsivri ve siklıkla mukrolu.

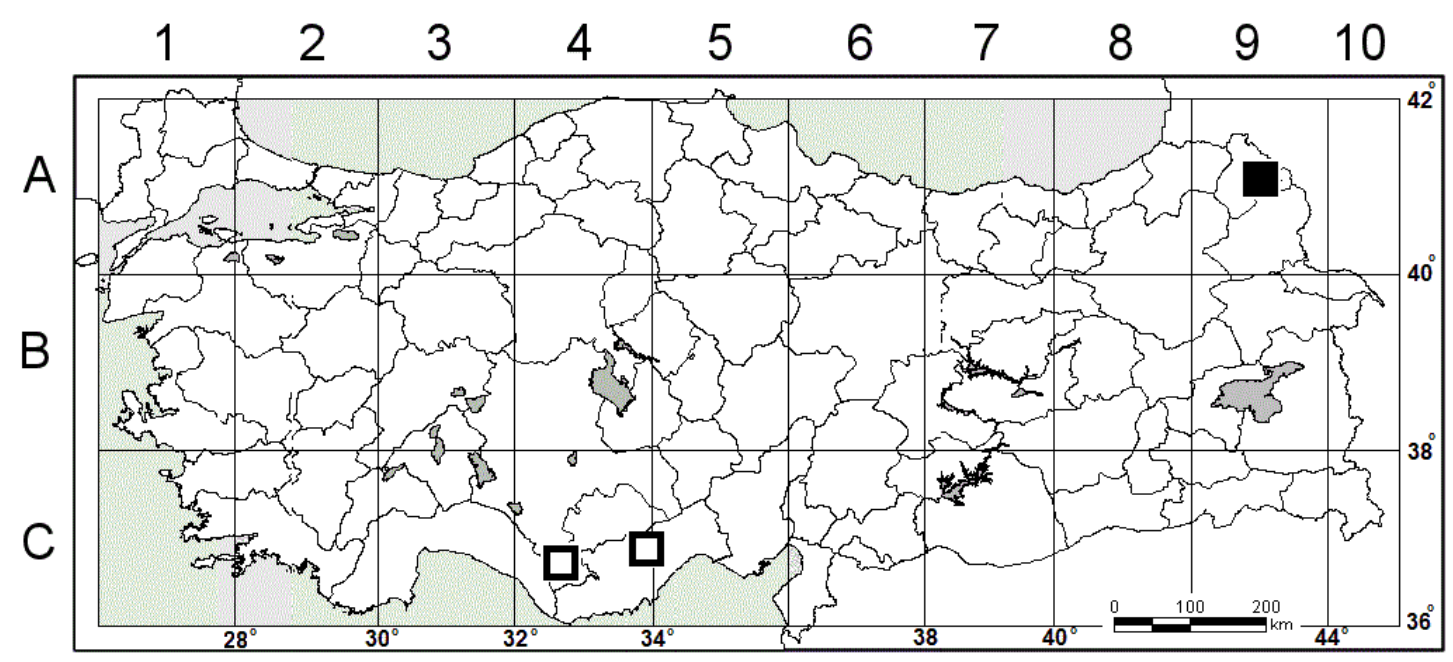

Şekil 3. Dianthus azkurensis (ロ) ve D. micranthus (ם)'un Türkiye'deki yayılışı. 
Çizelge 1. Dianthus azkurensis ve D. micranthus'un ayırtedici morfolojik karakterleri.

\begin{tabular}{|l|l|l|}
\hline Karakter & D. azkurensis & D. micranthus \\
\hline Alt yaprak & erken kuruyucu/dökülücü ve 30-50 mm boyunda & kalıcı ve 8-20 mm boyunda \\
\hline Diş kaliks pulu boyu & $3.5-5.5 .(-8) \mathrm{mm}$ & $2.2-4 \mathrm{~mm}$ \\
\hline Kaliks pulu kılçı̆̆ & tüm pulun 1/4-1/2'si kadar & tüm pulun $1 / 8-1 / 4$ 'ü kadar \\
\hline Kaliks boyu & $8.5-11 \mathrm{~mm}$ & $7-9 \mathrm{~mm}$ \\
\hline Petal boyu & $12-16 \mathrm{~mm}$ & $10-13 \mathrm{~mm}$ \\
\hline Petal kılavı boyu & $8-10 \mathrm{~mm}$ & $6.5-7.5 \mathrm{~mm}$ \\
\hline Tohum boyu & $1.5-2.2 \mathrm{~mm}$ & $2.5-3.5 \mathrm{~mm}$ \\
\hline
\end{tabular}

Petal 21-42 mm; aya kuneat, eliptik veya tersyumurtamsı, 6.5-15 × 4-9 $\mathrm{mm}$, tüm petalin 1/3'ü kadar, tamamı kaliksin dışında, beneksiz veya tabanda benekli, barbulat, beyaz veya pembe, kenar yandan uca kadar 7-23-dişli, dişler ayanın 1/4-1/2'si, kadar, 1.6-4.5 mm boyunda; kılav 15-28 × 1.2-2 mm, yaka eni kılav eninin en fazla $1 / 5$ 'i kadar. Meyve kaliksten kısa veya biraz uzun. Tohumlar eliptik veya paralel-eliptik, siyah, 2-3.7 × 1-1.5 mm.

1. Kaliks pulları (4-)6(-8) adet, gevşek ve düzensiz kiremitsi dizilmiş; en içtekiler kılçıklı veya kuspisli, kılçık veya kuspis tüm pulun 1/5-1/3'ü kadar, kenarları 0.4-0.6 mm eninde zarsi; petal (21-)26-30(-35) mm, aya $6.5-8.5(-10)$ $\mathrm{mm}$ boyunda subsp. nassireddini

-. Kaliks pulları (4-)6-12(-14) adet, sıkı ve düzenli kiremitsi dizilmiş; en içtekiler mukrolu, kenarları c. $0.3 \mathrm{~mm}$ eninde zarsi; petal $32-42 \mathrm{~mm}$, aya $10-15 \mathrm{~mm}$ boyunda 2

2. Orta yaprak kın boyu eninin en fazla 1.5 katı kadar; en dış kaliks pulları uca doğru silik 3-7-damarlı; en içtekiler uca doğru silik 7-15-damarlı, 3-3.8 mm eninde; kaliks dişleri 7-11-damarlı; tohumlar 2-2.5 mm boyunda (Türkiye'nin kuzeydoğusu) subsp. orientalis

-. Orta yaprak kın boyu eninin 2-3 katı kadar; en dış kaliks pulları uca doğru silik 1-3-damarlı; en içtekiler uca doğru silik 5-9-damarlı, 2.2-2.7 mm eninde; kaliks dişleri 5-7-damarlı; tohumlar 2.8-3.4 mm boyunda subsp. aphanoneurus subsp. orientalis, Şekil 4.

Holotypus [Gürcistan]: "in Iberia, circa Tiflisium, locis cretaceis, Adams s.n. (LE).

Syn.: Dianthus fimbriatus M.Bieb., in Bieberstein, Fl. Taur.-Caucas. 1: 332 (1808)! Holotypus: [Gürcistan] "ex agro Tiflisiensis, ?.?.1802, Bieberstin s.n. (LE; E'deki resim, E00301879-foto!).

Chiçeklenme: Haziran-Ă̆ustos. Habitat: Taşlı yamaçlar, kayalıklar, çalılık, 145-2050 m.

Gürcistan, Ermenistan, İran, Türkiye. A8 Erzurum: İspir-Yusufeli arası (Çoruh yolu), $850 \mathrm{~m}$, kayalıklar, 2.7.2013, Hamzaoğlu 6797 \& Koç (GAZI!); Artvin: Yusufeli, Çeltikdüzü-Dokumacılar arası, $750 \mathrm{~m}$, çalılık, 4.6.2008, Hamzaoğlu 5091 (ANK!);

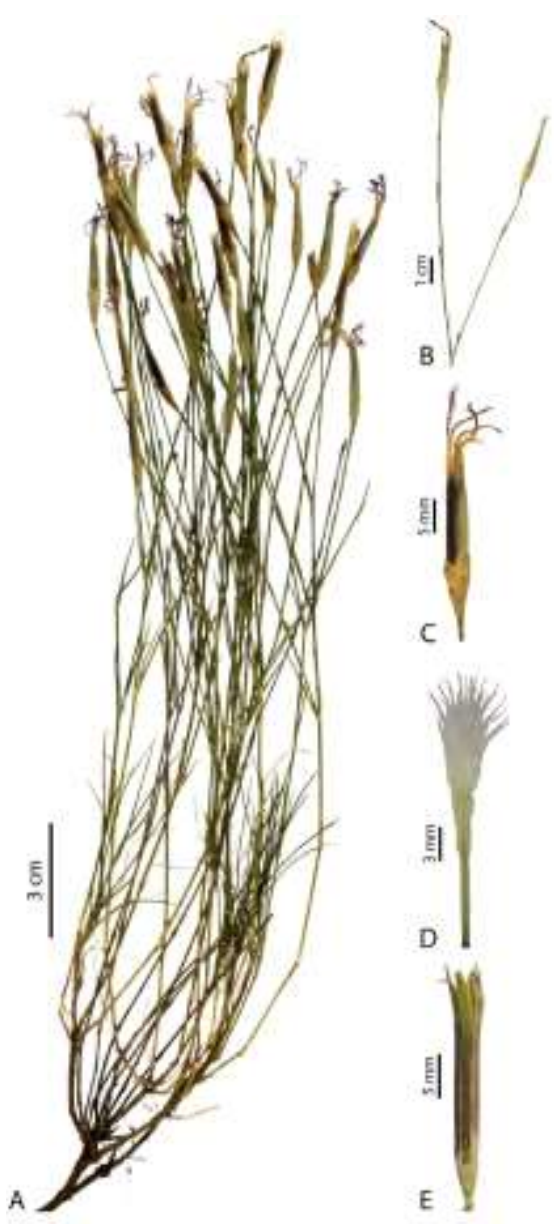

Şekil 4. Dianthus orientalis subsp. orientalis. A- Habit, B- Çiçekdurumu, C- Çiçek, D- Petal, EMeyve.

Yusufeli-Öğdem arası, 1235 m, 28.7.2010, kayalıklar, Hamzaoğlu 6055, Budak \& Koç (GAZI!); aynı yer, Yaylalar-Körahmet arası, verici civarı, $2050 \mathrm{~m}$, 21.8.2008, taşlı yamaçlar, Hamzaoğlu 5362 \& Aksoy (HUB!); Yusufeli-Artvin arası, Yusufeli çıkışı, suçatına varmadan, $545 \mathrm{~m}$, kayalıklar, 3.7.2013, Hamzaoğlu 6799 \& Koç (ANK!); aynı yer, Havuzlu köyü civarı, 620 $\mathrm{m}$, taşlı yerler, 3.7.2013, Hamzaoğlu 6802 \& Koç (HUB!); Borçka, Demirciler köyü çıkışı, $145 \mathrm{~m}$, kayalık, 23.7.2013, Hamzaoğlu 6905 \& Koç (GAZI!); A9 Erzurum: Şenkaya, Akşar köyü çıkışı, 1305 m, kayalık, 6.8.2014, Hamzaoğlu 7147 \& Koç (GAZI!); Oltu-Olur arası, Kaledibi köyü çıkışı, 1050 m, 25.8.2012, kayalıklar, Hamzaoğlu 6637 \& Koç (ANK!); Artvin: 
Ardanuç girişi, Cehennem deresi civarı, $510 \mathrm{~m}$, 3.7.2013, kalker kayalıklar, Hamzaoğlu 6803, Aksoy \& Koç (GAZI!); Şavşat, Demirkapı köyü yol ayrımı, 880 m, kayalık, 23.7.2013, Hamzaoğlu 6906 \& Koç (ANK!). subsp. nassireddini (Stapf) Rech.f., in Pl. Syst. Evol. 151: 292 (1986), Şekil 5.

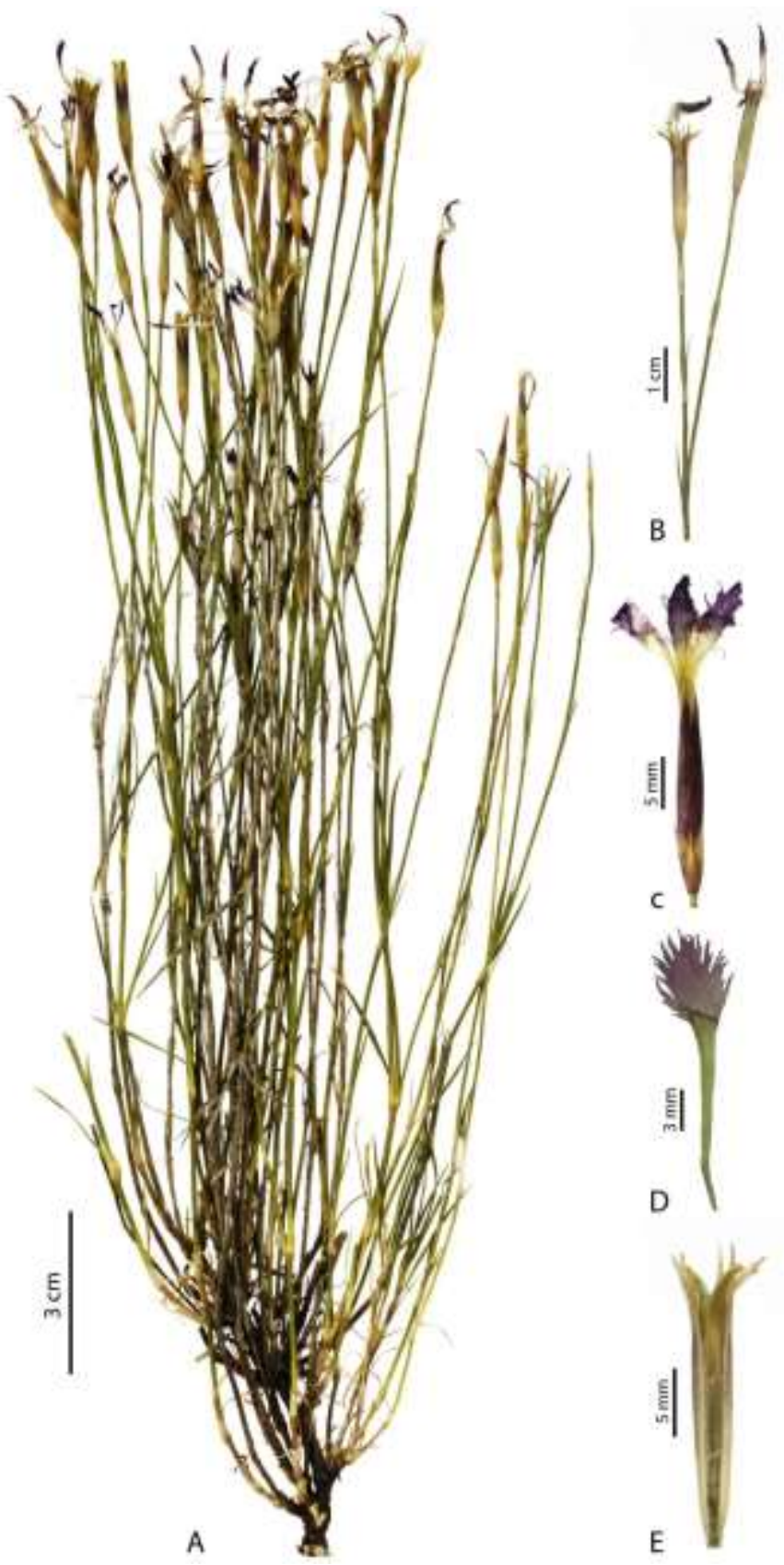

Şekil 5. Dianthus orientalis subsp. nassireddini. AHabit, B- Çiçekdurumu, C- Çiçek, D- Petal, EMeyve

Holotypus [İran]: "in fissures rupium montis Elwend supra Gendjname”, 10.6.1882, Th.Pichler s.n. (WU).

Syn: Dianthus nassireddini Stapf, in Stapf, Denkschr. Kaiserl. Akad. Wiss., Wien. Math.-Naturwiss. Kl. 51: 279 (1886a)!; D. fimbriatus M.Bieb. var. brachyodontus Boiss. \& A.Huet in Boissier, Diagn. Pl. Orient. 2(5): 53
(1856)! Holotypus: [Türkiye. A8 Erzurum] "circa Tortum, 4000-5000' [c. 1500-1900 m], ?.6.1853, H.Pavillon s.n. (G, G00226692-foto!); D. dumulosus Boiss. \& A.Huet in Boissier, Diagn. Pl. Orient. 2(5): 53 (1856)! Holotypus: [Türkiye. A8 Erzurum] "circa Tortum, 4000-5000' [c. 1500-1900 m], ?.6.1853, H.Pavillon s.n. (K, K000725529-foto!): D. var. macropetalus Boiss., Fl. Orient. Suppl.: 77 (1888)! Holotypus: [İran] "in rupestribus M. Schahu et Avroman, 8000-9000' [3000-3400 m], ?.7.1867, C.Haussknecht s.n. (G, G00226691-foto!); D. orientalis Adams subsp. macropetalus (Boiss.) Rech.f. in Pl. Syst. Evol. 151: 289 (1986).

Ciçeklenme: Haziran-Ağustos. Habitat. Taşlı yamaçlar, kayalıklar, 1270-3000 m.

İran-Turan elementi. İran, Irak, Ermenistan, Türkiye. A7 Giresun: Şebinkarahisar-Giresun arası, Aslanyurdu Deresi, 1330 m, 23.8.2012, kayalıklar, Hamzaoğlu 6616 \& Koç (GAZI!); aynı yer, $1470 \mathrm{~m}$, 13.6.2006, kayalıklar, Hamzaoğlu 3892, Aksoy \& Budak (ANK!); Şebinkarahisar, Hacıömer köyü kuzeyi, 1270 m, 13.6.2006, taşlı yamaçlar, Hamzaoğlu 3900, Aksoy \& Budak (HUB!); Gümüşhane: KaleNazlıçayır köyleri arası, c. 1700 m, 18.6.2003, Hamzaoğlu 3473 (GAZI!); Torul, Ekinciler-Arpacık arası, 1690 m, 14.7.2007, kayalık yerler, Hamzaoğlu 4809 \& Budak (ANK!); Sivas: Zara-Suşehri arası, Karalar köyü yol ayrımı, 1450 m, 9.8.2009, kayalıklar, Hamzaoğlu 5556, Budak \& Koç (HUB!); A8 Bayburt: Bayburt'un güneydoğusundaki tepeler, 1500-1750 m, 23.6.2002, bozkır, Hamzaoğlu 2922 (GAZI!); BayburtAşkale arası, Kop Dağı, 2140 m, 24.7.2010, kayalıklar, Hamzaoğlu 5912, Budak \& Koç (ANK!); Erzurum: Erzurum-İspir arası, $2010 \mathrm{~m}$, akıntılı kayalık yerler, 2.7.2013, Hamzaoğlu 6789 \& Koç (HUB!); B6 Kayseri: Pınarbaşı, Aşağıbeyçayırlı-Yukarıbeyçayırlı arası, 1755 m, 25.6.2010, akışkan taşlı yerler, Hamzaoğlu 5698, Budak \& Koç (GAZI!); Sarız, Kırkısrak köyü, Binboğa Dağları, Salıngaç mevki, 2100 m, 16.7.2009, otlu yamaçlar, Koç 740, Hamzaoğlu \& Budak (ANK!); Tomarza, Arslantaş köyü, yol ayrımından c. $1 \mathrm{~km}$ sonra, $1600 \mathrm{~m}$, 12.7.2012, kalker kayalıklar, Hamzaoğlu 6472 \& Koç (HUB!); Malatya: HekimhanKurşunlu arası, 1810 m, 26.6.2010, taşlı yerler, Hamzaoğlu 5768, Budak \& Koç (GAZI!); Sivas: Divriği, Höbek köyü güneybatısı, 1860 m, 2.7.2008, bozkır, Hamzaoğlu 5185 \& Aksoy (ANK!); Gürün, BaşörenHaticepınar köyleri arası, 1910 m, 13.7.2008, kalker kayalıklar, Hamzaoğlu 5219 \& Aksoy (ANK!); B7 Erzincan: Ergan Dağı Kayak Merkezi, Öğletaşı Tepe, 2250 m, kalker kayalık, 6.7.2014, Hamzaoğlu 7062 \& Koç (HUB!); Refahiye-İmranlı arası, Kızıldağ Geçidi yakını, $1770 \mathrm{~m}$, akıntılı yamaçlar, 4.8.2014, Hamzaoğlu 7124 \& Koç (GAZI!); Tunceli: TunceliPülümür arası, Kırmızıköprü civarı, 1350 m, 9.6.2011, kayalıklar, Koç 1316 \& Hamzaoğlu (GAZI!); B8 Erzurum: Çat, Muratçayırı-Aşağıkumaşlı köyleri 
arası, $1700 \mathrm{~m}$, 14.6.2007, vadi içi, kayalıklar, Hamzaoğlu 4615 \& Aksoy (ANK!); Çat-Bingöl arası, Kirişli Geçidi civarı, 2320 m, 16.7.2007, Hamzaoğlu 4847, Aksoy \& Budak (GAZI!); Palandöken Dağı, zirveye giden yol, $2600 \mathrm{~m}, 2.7 .2006$, otlu yamaçlar, Hamzaoğlu 4138 \& Budak (HUB!); Muş: Muş-Üçevler Köyü arası, Elmani Yaylası civarı, 2410 m, 28.8.2012, taşlı yamaçlar, Hamzaoğlu 6665 \& Koç (ANK!); Varto, Çaylar, Tuzla köyü üstü, 2520 m, Astragalus'lu yamaçlar, 29.8.2012, Hamzaoğlu 6672 \& Koç (GAZI!); B9 Ağrı: Ağrı-Hamur arası, Hamur yakınları, 1680 m, akıntılı yamaçlar, 5.7.2013, Hamzaoğlu 6827 \& Koc (ANK!); Eleşkirt-Horasan arası, Tahir köprüsü, vadi girişi, $2005 \mathrm{~m}$, volkanik yamaçlar, 4.7.2014, Hamzaoğlu 7046 \& Koç (HUB!); Van: Gevaş, Artos Dağının Gevaş'a bakan yamaçları, $2600 \mathrm{~m}$, kalker yamaçlar, 14.7.2009, Koç 672 \& Hamzaoğlu (GAZI!); Gevaş-Tatvan arası, Pelli Dağı, 2600-3000 m, taşlı yamaçlar, 13.8.2013, Hamzaoğlu 6959 (ANK!); Gürpınar-Çatak arası, Ganispi Şelalesi üstü, $1750 \mathrm{~m}$, 1.7.2006, Hamzaoğlu 4098 \& Budak (HUB!); C5 Niğde: Çamardı, Demirkazık köyü üstü, 1475 m, 11.7.2012, kayalıklar, Hamzaoğlu 6451, Aksoy \& Koç (GAZI!); aynı yer, Narpız Boğazı girişi, 2485 m, 25.7.2012, taşlı yamaçlar, Hamzaoğlu 6520, Aksoy \& Koç (ANK!); Ulukışla, Bolkar Dağı, Maden köyü üstü, 1825 m, 19.7.2005, kayalıklar, Budak 1764, Hamzaoğlu \& Aksoy (HUB!); C10 Hakkâri: $30 \mathrm{~km}$ from Başkale to Hakkâri, 1900 m, on rocks, 10.6.1966, P.H.Davis 44676 (E-foto!); Cilo Dağı, above Diz Deresi, 3000 m, 7.8.1954, P.H.Davis 23939 (E-foto!).

subsp. aphanoneurus Rech.f., in Pl. Syst. Evol. 151: 290 (1986), Şekil 6.

Holotypus: [İran] Luristan, M. Sawers, 9000' [c. 3400 m], ?.7.1868, C.Haussknecht s.n. (W, W0026404-foto!).

Syn.: Dianthus aphanoneurus (Rech.f.) M.L.Kuzmina, Bot. Zhurn. (Moscow \& Leningrad) 81: 82 1996).

Ciçeklenme: Temmuz-Ă̆ustos. Habitat: Kumlu taşl yamaçlar, kayalıklar, orman açıklı̆̆g, 980-1300 m.

İran-Turan elementi. Ermenistan, İran, Türkiye. B6

Kayseri: Sarız, Kıskaçlı köyü, KayseriKahramanmaraş yolu, dinlenme tesisleri civarı, 1830 m, akışkan kalker kayalıklar, 8.8.2018, Hamzaoğlu \& Koç 3491 (GAZI!, ANK!, HUB!); Malatya: Darende, Aşağıulupınar köyü güneyi, $1300 \mathrm{~m}$, kumlu taşlı yamaçlar, 12.7.2010, Koç 1245 \& Hamzaoğlu (GAZI!, ANK!, HUB!); aynı yer, taşlı kumlu yamaç, 25.7.2013, Hamzaoğlu 6947 \& Koç (GAZI!); C5 Mersin: Toroslar, Arslanköy-Mersin arası, c. $20 \mathrm{~km}, 980 \mathrm{~m}$, orman açıklığı, 19.8.2013, Hamzaoğlu 6988 \& Koç (GAZI!, ANK!, HUB!).

Dianthus orientalis, "Flora of Turkey and the East Aegean Islands" adlı eserde monotipik bir tür olarak verilmiş olmasına rağmen, daha sonra yapılan detaylı çalışmalarda varyete veya alttürlere ayrılan politipik bir tür olarak değerlendirilmiştir.

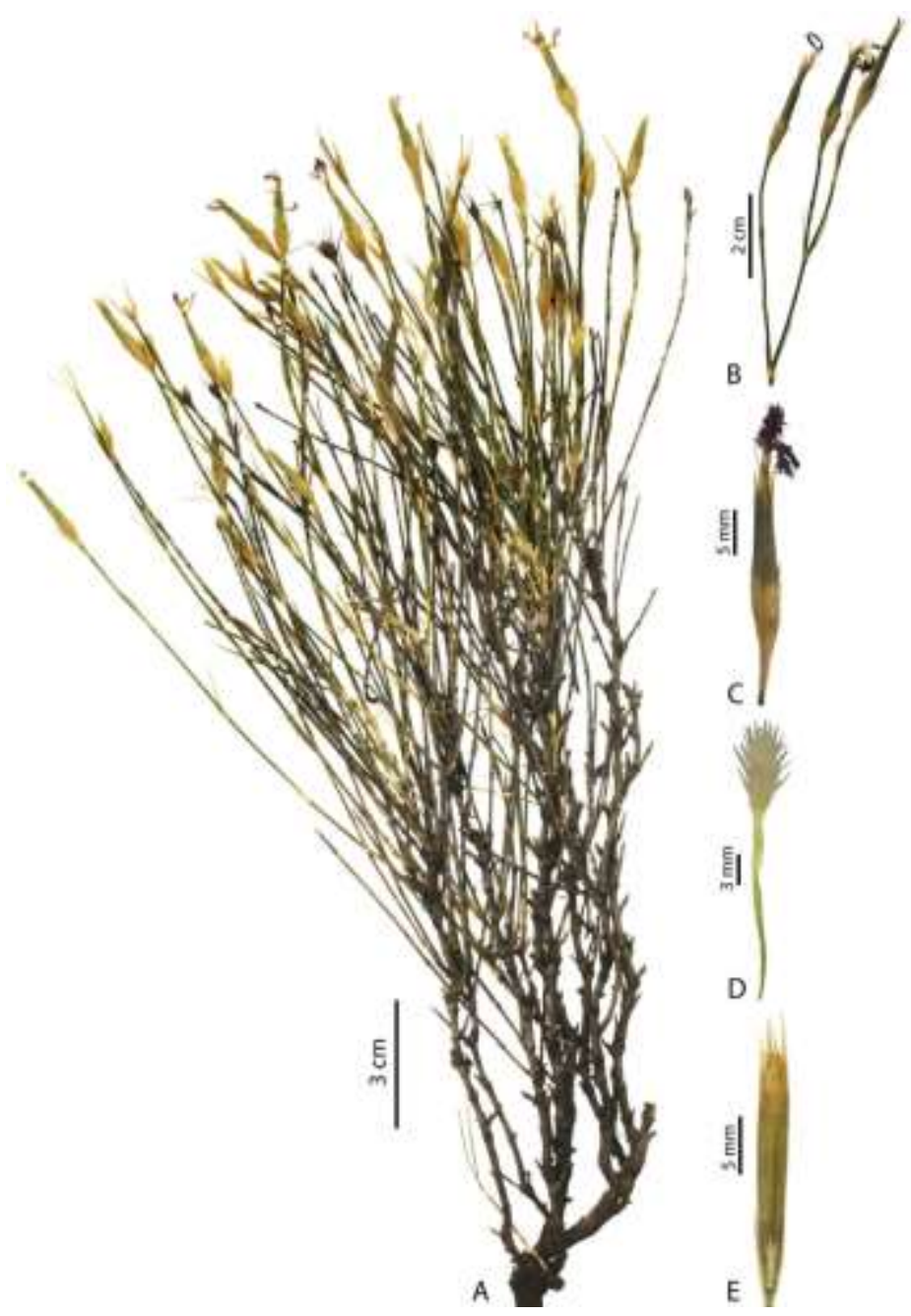

Şekil 6. Dianthus orientalis subsp. aphanoneurus. AHabit, B- Çiçek durumu, C- Çiçek, D- Petal, E- Meyve

Örneklerin ve ilgili literatürlerin incelenmesi sonucu, bu türaltı taksonlardan 3'ünün Türkiye'de yetiştiği tespit edilmiştir (Reeve, 1967; Dalc1, 1986; Rechinger, 1988). Buna göre, Dianthus orientalis (subsp. orientalis) sadece Türkiye'nin kuzeydoğusunda yetişen bir taksondur (Şekil 7). Subsp. nassireddini ve subsp. aphanoneurustan kaliks pulu sayısı, kaliks pulu damar sayısı, kaliks pulu genişliği, kaliks dişi damar sayısı, petal ve aya boyu gibi karakterlerle ayrilır.

Dianthus orientalis, Doğu Anadolu Dianthus türleri üzerine yapılan çalışmada var. orientalis ve var. brachyodontus Boiss. \& A.Huet şeklinde 2 varyete olarak değerlendirilmiştir. Bunlardan var. brachyodontus, eserde " $D$. orientalis var. brachyodontus (Boiss. \& A.Huet) Bornm.” şeklinde gösterilmiştir. Ancak "Bornmüller" tarafından $D$. fimbriatus'tan $D$. orientalise yapılan bu aktarmaya ait herhangi bir makaleye ulaşılamamıştır. Var. brachyodontus, "Flora Iranica" da olduğu gibi bu makalede de subsp. nassireddini altında sinonim olarak kabul edilmiştir (Dalcı, 1986; Rechinger, 1988). 
Subsp. nassireddini, diğer alttürlerden kaliks boyu ile kaliks pulunun sayısı, şekli ve yapısı gibi karakterlerle ayrılmıştır. "Flora of Turkey and the East Aegean Islands"da bu karakterler oldukça geniş verilmiş ve $D$. orientalis monotipik bir tür olarak tanıtılmıştır (Reeve, 1967; Rechinger, 1988). Dianthus orientalis subsp. nassireddini özellikle Anadolu Çaprazının doğusunda yaygın olarak yetişir (Şekil 7).

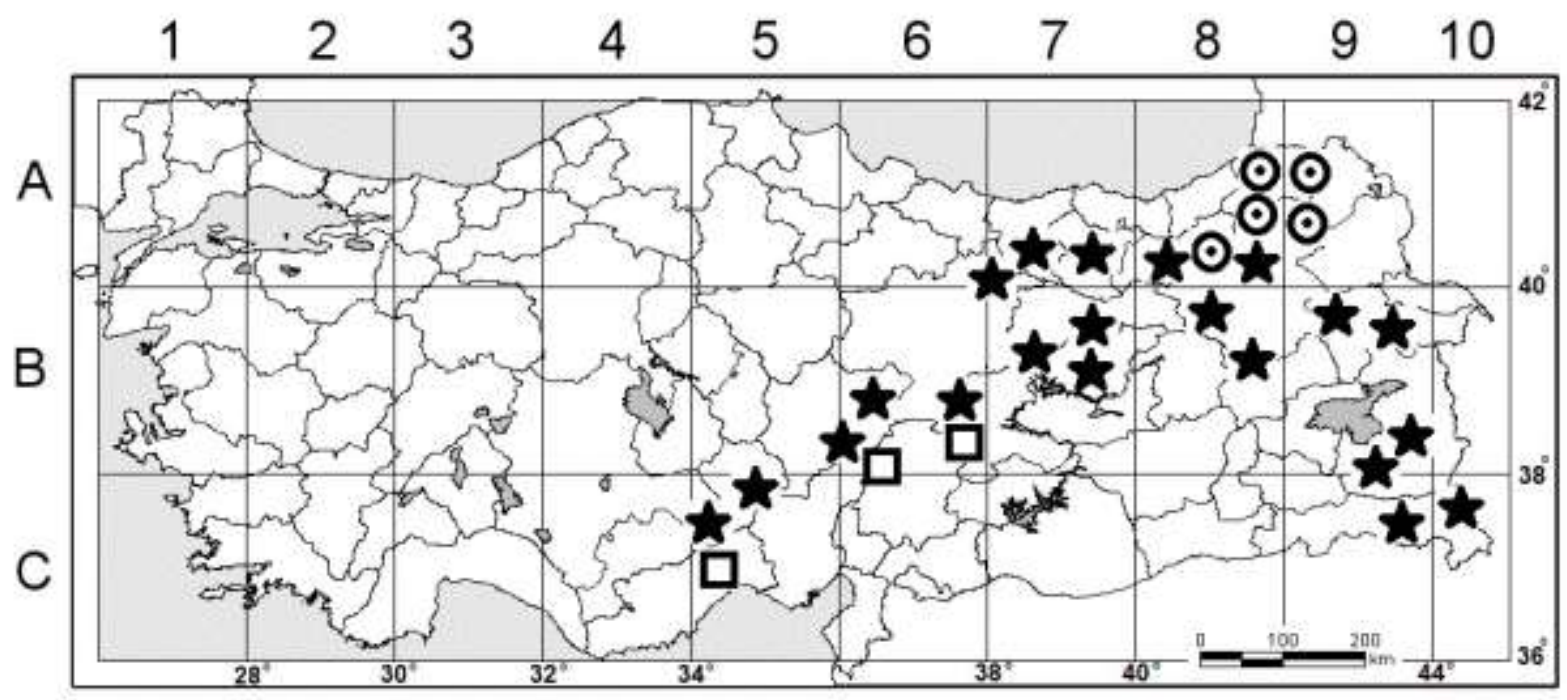

Şekil 7. Dianthus orientalis subsp. orientalis $(\odot)$, D. orientalis subsp. nassireddini $(\star)$ ve D. orientalis subsp. aphanoneurus (ם)'un Türkiye'deki yayılışı

Detaylı inceleme ve kaynak taranması sonucu, Kayseri, Malatya ve Mersin'den toplanan örneklerin (Koç 1245 ve 3941, Hamzaoğlu 6947 ve 6988) "Flora Iranica"ya göre İran'ın güneybatısından bilinen Dianthus orientalis subsp. aphanoneurus taksonuna ait olduğuna karar verilmiştir (Şekil 7). "Flora of Turkey and the East Aegean Islands" adl eserde, $D$. orientalis türüne ait dip notta Nur Dağlarında (Amanos) 10 veya daha fazla kaliks puluna sahip bazı örneklerin varlığından bahsedilmiştir. Ancak bu örnekler subsp. aphanoneurus yerine $D$. sinaicus Boiss.'e benzetilmiştir. D. sinaicus, Nur Dağlarından kuş uçuşu yaklaşık $600 \mathrm{~km}$ güneyde olan Sina yarımadasında yetişir. Kayalık alanları tercih eden türde kaliks pulu sayısı 10'dur ((10-)12-14(-16) değil) ve petal ayası ebarbulattır (barbulat değil) (Post, 1932; Reeve, 1967; Rechinger, 1988). Türkiye için yeni kayıt olan $D$. orientalis subsp. aphanoneurus ile subsp. nassireddini, özellikle kaliks pulu sayısı bakımından farklılık gösterir (Tablo 2).

Tablo 2. Dianthus orientalis subsp. aphanoneurus ve D. orientalis subsp. nassireddininin ayırtedici morfolojik karakterleri

\begin{tabular}{|l|l|l|}
\hline Karakter & D. orientalis subsp. aphanoneurus & D. orientalis subsp. nassireddini \\
\hline Alt yaprak & erken kuruyucu/dökülücü & kalıcı \\
\hline Orta yaprak kını & boyu eninin 2-3 katı kadar & boyu eninin en fazla 1.5 katı kadar \\
\hline Kaliks pulu sayısı & $\left(10^{-}\right) 12-14(-16)$ adet & $((4-) 6(-8)$ adet \\
\hline
\end{tabular}

\section{TEŞEKKÜR}

Türkiye florası için yeni kayıt olarak tanıtılan Dianthus azkurensis ve Dianthus orientalis subsp. aphanoneurus, TÜBİTAK tarafindan desteklenen "Türkiye Dianthus cinsi (Caryophyllaceae) revizyonu" adlı ve KBAG-111T873 kodlu proje kapsamında toplanmıştır. Maddi desteği nedeniyle TÜBİTAK'a teşekkür ederiz. Ayrıca, bazı Dianthus herbaryum örneklerini incelediğimiz GAZI (Gazi Üniversitesi), ANK (Ankara Üniversitesi ve HUB (Hacettepe Üniversitesi) herbaryum sorumluları ile bazı Dianthus örneklerine ait detaylı fotoğrafları incelediğimiz $\mathrm{E}$ (Edinburgh) ve K (Kew) sanal herbaryum sorumlularına teşekkür ederiz.

\section{KAYNAKLAR}

Bacchetta G, Brullo S, Casti M, Giusso del Galdo GP 2010. Taxonomic revision of the Dianthus sylvestris group (Caryophyllaceae) in central-southern Italy, Sicily and Sardinia. Nordic Journal of Botany, 28(2): 137-173.

Bittrich V 1993. Caryophyllaceae. (The Families andGenera of Vascular Plants, Magnoliid, Hamamelid, and Caryophyllid Families, Springer, Berlin: Ed. Kubitzki K, Rohwer J, Bittrich V) Vol. 2: 206-236.

Boissier E 1867. Flora Orientalis. H. Georg, Geneva and Basel, Vol. 1: 479-516.

Constantinidis T 1999. Dianthus haematocalyx subsp. 
phitosianus (Caryophyllaceae), a new serpentine endemic from Greece. Phyton, 39(2): 277-291.

Dalcı M 1986. Morphological studies and new synonyms of some species in Dianthus L. DOĞA Turkish Journal of Biology (Botany), 10(2): 138-159.

Dequan L, Turland NJ 2001. Dianthus L. (Flora of China, Science Press and Missouri Botanical Garden Press, Beijing and St. Louis: Ed. Jussie AL) Vol. 6: 102-107.

Hamzaoğlu E, Koç M, Aksoy A 2014. A new pricking Carnation (Caryophyllaceae) grows on tuff from Turkey: Dianthus aculeatus sp. nov. Biological Diversity and Conservation, 7(2): 159-162.

Hamzaoğlu E, Koç M 2015. Dianthus burdurensis (Caryophyllaceae), a new species from Southwestern Turkey. Phytotaxa, 233(2): 196-200.

Hamzaoğlu E, Koç M, Büyük İ 2018. Two new spiny species of Dianthus (Caryophyllaceae) from Turkey. KSU Journal of Agriculture and Nature, 21(4): 545554.

Hamzaoğlu E, Koç M, Aksoy A 2015a. Dianthus aticii, a new species from Turkey (Caryophyllaceae). Phytokeys, 48: 21-28.

Hamzaoğlu E, Koç M, Büyük İ, Aksoy A, Soydam Aydın S 2015b. Presence of Dianthus roseoluteus Velen. (Caryophyllaceae) in Turkey and a new species: Dianthus macroflorus Hamzaoğlu. Systematic Botany, 40(1): 208-213.

Hamzaoğlu E, Koç M, Büyük İ, Aksoy A, Soydam Aydin S 2015c. A new serpentine-adapted carnation (Caryophyllaceae) from Turkey: Dianthus serpentinus sp. nov. Nordic Journal of Botany, 33(1): 57-63.

İlçim A, Behçet L, Mükemre M 2013. Dianthus vanensis (Caryophyllaceae), a new species from
Turkey. Turkish Journal of Botany, 37(2): 219-224.

Mabberley DJ 2008. Mabberley's Plant-book: a portable dictionary of plants, their classification and uses. Cambridge University Press, Cambridge, third edition, 1021s.

Özhatay N, Kültür Ş 2006. Check-list of additional taxa to the supplement Flora of Turkey III. Turkish Journal of Botany, 30(4): 281-316.

Post GE 1932. Flora of Syria, Palestina and Sinai. Syrian Protestant College, Beirut, Vol. 1: 154-160.

Rechinger KH 1988. Dianthus L. (Flora Iranica, Akademische Druck-u Verlagsanstalt, Graz: Ed. Rechinger KH) Caryophyllaceae II: 128-188.

Reeve H 1967. Dianthus L. (Flora of Turkey and the East Aegean Islands, Edinburgh University Press, Edinburgh: Ed. Davis PH) Vol. 2: 99-131.

Schischkin BK 1985. Dianthus L. (Flora of the U.S.S.R., Bishen Singh Mahendra Pal Singh \& Koeltz Scientific Books, Dehra Dun: Ed. Schischkin BK) Vol. 6: 611-654.

Thorne RF, Reveal JL 2007. An updated classification of class Magnoliopsida (Angiosperm). The Botanical Review, 73(2): 67-182.

Vaezi J, Behroozian M, Memariani F, Joharchi MR 2014. Dianthus pseudocrinitus (Caryophyllaceae), a new species from Northeast of Iran identified by morphological and molecular data. Phytotaxa, 156(2): 59-73.

Valente LM, Savolainen V, Vargas P 2010. Unparalleled rates of species diversification in Europe. Proceedings of The Royal Society B: Biological Sciences, 277(1687): 1489-1496.

Williams FN 1893. A monograph of the genus Dianthus. Botanical Journal of the Linnean Society, 29(203): 346-378. 